\title{
Preventive and Therapeutic Effects of Chlorhexidine Containing Varnish on Candida Biofilm
}

\author{
KAROLY MENSCH ${ }^{1,2}$, JULIA PONGRACZ ${ }^{3}$, ADAM NAGY ${ }^{4}$, KATALIN KRISTOF $^{3}$, ANAMARIA BECHIR ${ }^{5 *}$, MARIANA PACURAR, \\ GABOR NAGY ${ }^{1}$ \\ ${ }^{1}$ Semmelweis University, Faculty of Dentistry, Department in Community Dentistry, 26 Ulloi Blvd, 1085 Budapest, Hungary \\ ${ }^{2}$ Semmelweis University, Faculty of Dentistry, Department of Oral Diagnostics, 26 Ulloi Blvd, 1085 Budapest, Hungary \\ ${ }^{3}$ Semmelweis University, Faculty of Medicine, Department of Laboratory Medicine, 26 Ulloi Blvd, 1085 Budapest, Hungary \\ ${ }^{4}$ Semmelweis University, Faculty of Medicine, 26 Ulloi Blvd, 1085 Budapest, Hungary \\ ${ }^{5}$ Titu Maiorescu University of Bucharest, Faculty of Dental Medicine, 67A Gh. Petrascu Str., 031593, Bucharest, Romania \\ ${ }^{6}$ University of Medicine and Pharmacy Tirgu Mures, Faculty of Dental Medicine, 38 Gheorghe Marinescu Str., 540139, Tirgu \\ Mures, Romania
}

\begin{abstract}
The aim of the study was to assess the preventive and therapeutic efficacy of a sustained-release varnish (SRV) Cervitec Plus ${ }^{\circledR}$ compared with Corsodyl $₫$ mouthwash against Candida albicans, Candida parapsilosis, Candida tropicalis and Candida glabrata on acrylate surfaces. Nystatin as the positive control, and sterile saline solution as the negative control, were used. To examine the preventive efficacy of the agents, Candida biofilms were developed in Sabouraud liquid medium, and mature biofilms were treated with the antifungal. To investigate the preventive efficacy, the agents were applied to the wells of the plates and the biofilms were grown in the treated wells. To determine the metabolic activity of biofilms, the XTT-assay was used. In the case of $C$. albicans and C. parapsilosis, the most effective therapeutic product was Corsodyl ${ }^{\circledR}$ followed by Cervitec Plus ${ }^{\circledR}$ and Nystatin. The differences were statistically significant when Corsody ${ }^{\circledR}$ was compared to Nystatin $(p \leq 0.05)$. In relation to C. tropicalis and C. glabrata, Corsody $\left.\right|^{\circledR}$ showed the greatest therapeutic efficacy followed by Nystatin and Cervitec Plus ${ }^{\circledR}$. For both species, the results of Corsody ${ }^{\circledR}$ and Cervitec Plus ${ }^{\circledR}$ showed statistically significant difference $(p \leq 0.05)$. The most effective preventive product for each examined Candida species was Nystatin, followed by Cervitec Plus ${ }^{\circledR}$ and Corsody ${ }^{\circledR}$. Significant differences were between Nystatin and Corsody ${ }^{\circledR}(p \leq 0.05)$. The preventive and therapeutic efficacy of Cervitec Plus ${ }^{\circledR}$ and Corsodyl ${ }^{\circledR}$, against the examined Candida species, was detected. Because of the disadvantageous side-effects of Nystatin, Cervitec Plus ${ }^{\circledR}$ can be considered as an alternative preventive agent against Candida species.
\end{abstract}

Keywords: Candida biofilm, denture stomatitis, sustained release varnish

Oral candidiasis is a frequent opportunistic infection affecting the oral mucosa, caused by overgrowth of several Candida species [1], the mostcommon is Candida albicans $[2,3]$, C. albicans may be commensal in 30 to $45 \%$ of healthy adult mouths [4,5]. It can induce opportunistic infections when appropriate predisposing factors exist (e.g. changes in the oral mucosal environment or in the immune status of the host).

Dental plaque is a multispecies biofilm of different microorganisms (bacteria and yeasts) growing as an ecosystem on soft and hard tissues in the oral cavity, including teeth, fillings, dentures. The Candida associated denture stomatitis is the most common form of oral candidosis [6-8].

Mechanical tooth cleaning (toothbrushing with toothpaste) is the most common and most effective form of individual oral hygiene. How ever, very often it is difficult to achieve a proper level of oral hygiene using only toothbrush. Patients' efforts could be compromised by the presence of inadequate physical ability (e.g. after severe operations, stroke, severe arthritis) so, the adjunctive use of an antiseptics and D or other chemical agents are also justified.

Chlorhexidine digluconate $(\mathrm{CHX})$ is still considered to be the leading antiseptic for combating oral biofilms [9]. The chemical structure of chlorhexidine digluconate is presented in figure 1 [10].

The mechanism of action of chlorhexidine digluconate is presented in figure 2 [11].

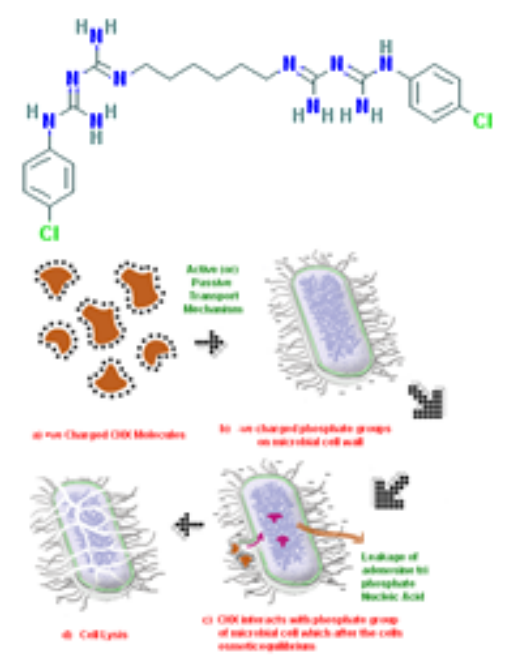

Fig. 1. Chemical structure of chlorhexidine digluconate

Fig. 2. Chlorhexidine digluconate: mechanism of action

Recently, local sustained-release varnishes (SRV) and gels have become available to eliminate cariogenic and periodontopathogenic bacteria. Local SRV extend the time when the active ingredients present in the oral cavity, thus enhancing their therapeutic effect. These varnishes and gels are mainly targeted against cariogenic bacteria and their antibacterial effects were extensively examined $[9,12]$, while their antifungal activity had not been analyzed before in details, except $\mathrm{CHX}$ solutions but usually on planctonic cells [13-16].

Biofilms are the immobilized cells in an organic polymer matrix of microbial origin. The ability to form biofilms, 
considered recently the attribute only for a few species is now seen as an attribute for almost all microorganisms [17]. Oral biofilms show different sensitivity toward antifungal agents compared to planktonic bacteria, so it is necessary to check the efficacy of already known antifungal (e.g.CHX) against Candida biofilms using proper microbiological methods [18].

The active ingredients of Cervitec Plus ${ }^{\circledR}$ varnish (Ivoclar VivadentAG, Schaan, Liechtenstein) (fig. 3), is a protective varnish containing chlorhexidine and thymol, which allows to a selectively control of germs and therefore reduce the caries risk factor, prevents and helps the treatment of gingivitis, protects teeth in situations where patients are incapable of carrying out proper oral hygiene [19].

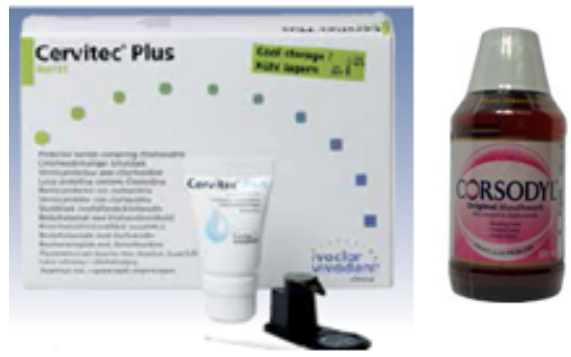

Fig. 4. Corsody ${ }^{\circledast} 2 \%$ mouthwash: manner of presentation

Fig. 3. Cervitec Plus ${ }^{\circledR}$ varnish: manner of presentation

Corsodyl $0.2 \%$ Mouthwash contain Chlorhexidine Digluconate $0.2 \% \mathrm{w} / \mathrm{v}$ (equivalent to Chlorhexidine Digluconate Solution $1.028 \% \mathrm{w} / \mathrm{w}$ ) and macrogolglycerol hydroxystearate. Is a clear to slightly opalescent, transparent solution, used for inhibition of the formation of dental plaque, in the treatment and prevention of gingivitis and in the maintenance of oral hygiene, particularly in situations where toothbrushing cannot be adequately employed (e.g. following oral surgery, in mentally or physically handicapped patients) [20].

The aim of our examination was to evaluate the therapeutic efficacy of Cervitec Plus ${ }^{\circledR}$ varnish (Ivoclar Vivadent AG, Schaan, Liechtenstein) against the biofilm of four different Candida species (Candida albicans, Candida parapsilosis, Candida tropicalis, and Candida glabrata) comparing to those of Corsodyl ${ }^{\circledR}$ mouthwash. Furthermore we tried to assess if Cervitec Plus ${ }^{\circledR}$ varnish can prevent biofilm formation of Candida species on acrylate surfaces compared with $0.2 \% \mathrm{CHX}$ containing mouthwash (Corsodyl ${ }^{\circledR}$-GlaxoSmithKline PLC, Brentford, London, United Kingdom).

\section{Experimental part}

\section{Materials and methods}

To evaluate the therapeutic efficacy of Corsodyl ${ }^{\circledR}$ and Cervitec Plus ${ }^{\circledR}$ varnish, Candida spp. were cultured for 48 hours in 24-well plates with each well containing a standard ( $6 \mathrm{~mm}$ in diameter and $1 \mathrm{~mm}$ thick) acrylic disk and $0.5 \mathrm{ml}$ of Sabouraud medium supplemented with $8 \%$ glucose. One isolate each of $C$. albicans, $C$. tropicalis, $C$. parapsilosis, and $C$. glabrata were tested. The isolates derived from clinical samples at Semmelweis University Clinical Microbiology Laboratory, and all proved to be biofilm producers in previous studies. After $48 \mathrm{~h}$ of incubation at $37^{\circ} \mathrm{C}$, the acrylic disks were washed in sterile saline, and the antifungal agents were applied to the biofilm coated disks (5 min each). Nystatin was used as positive control, and sterile saline as negative control. After treatment, the metabolic activity of the biofilms was measured by the 2,3-bis (2-methoxy-4- nitro-5-sulfophenyl)-5-[( phenylamino) carbonyl]-2H-tetrazolium hydroxide (XTT) assay. In short, $100 \mu \mathrm{L}$ of $0.5 \mathrm{mg} / \mathrm{ml}$ XTT was added to the wells containing the disk, and optical density (OD) was measured at $450 \mathrm{~nm}$ following incubation for $3 \mathrm{~h}$ at $37^{\circ} \mathrm{C}$. All tests were performed in triplicate.

For evaluation of the preventive effect of the antifungal products, the acrylic disks were first treated with the products then incubated with $C$. albicans, $C$. tropicalis, $C$. parapsilosis, and C. glabrata suspensions for 1 hour at $37^{\circ} \mathrm{C}$. After 1 hour, the disks were washed in saline to remove unattached yeast cells, followed by incubation in $0.5 \mathrm{ml}$ of Sabouraud medium containing $8 \%$ glucose. After $48 \mathrm{~h}$, the disks were washed and the metabolic activity of the biofilms was measured by the XTT method. The optical density (OD) was measured at $450 \mathrm{~nm}$.

To determine the percentual reduction of viable cells in the therapeutic and preventive trials the OD values of each chemical was compared to the OD value of negative control (saline solution).

To calibrate the XTT-assay the metabolic activity of certain Candida species of same germ count of $C$. albicans, C. parapsilosis, C. tropicalis and C. glabrata species was measured according to Kuhn: no outstanding difference was detectable in our experiment [21].

For the statistical analysis we used IBM SPSS statistics 23 software (IBM Corporation). The metabolic activity of Candida species was characterized with the mean and standard deviation of the percentual reduction of optical density. To determine the differences among the efficiency of solutions Kruskal-Wallis and Tukey post hoc tests were used by each Candida species and the statistical significance was accepted at $p<0.05$.

\section{Results and discussions}

Table 1 shows the the percentual reduction of optical density by each Candida spp. on the biofilm in the therapeutic effect trial. The label A, B, C means significant differences between the effects of the solution by each Candida ssp.

We found that the efficacies of the solutions were higher than $49 \%$ except in case of Cervitec Plus ${ }^{\circledR}$ and C. glabrata, where OD reduction was only $13 \%$. By all examined Candida species, the highest OD reduction was achieved by Corsodyl ${ }^{\circledR}$, which was between 95.24 and $97.54 \%$.

In the case of C. albicans and C. parpapsilosis, Corsodyl ${ }^{\circledR}$ was followed by Cervitec Plus ${ }^{\circledR}$ and Nystatin. By these species we found (table 1) significant differences between the efficiency of Corsodyl ${ }^{\circledR}$ and Nystatin $(p \leq 0.01)$. The effect of Cervitec Plus ${ }^{\circledR}$ was slightly better than that of Nystatin in case of C. albicans, and C. parapsilosis, the effect of Cervitec Plus ${ }^{\circledR}$ was weaker, but the differences were not statistically different compared to the two other solutions. Regarding C. tropicalis and C. glabrata the

\begin{tabular}{||l||c|c|c|c||}
\hline \multicolumn{1}{|c||}{\multirow{2}{*}{ Solutions }} & \multicolumn{4}{c||}{ Percentual reduction of OD (\%) } \\
& C. albicans & C. parapsilosis & C. tropicalis & C. glabrata \\
\hline \hline A. Corsodyl & $95.24 \pm 0.94^{\mathrm{C}}$ & $95.82 \pm 0.30^{\mathrm{C}}$ & $97.54 \pm 0.21^{\mathrm{B}}$ & $96.16 \pm 0.38^{\mathrm{B}}$ \\
\hline B. Cervitec Plus & $60.05 \pm 0.92$ & $66.60 \pm 0.68$ & $63.36 \pm 1.84^{\mathrm{A}}$ & $13.86 \pm 8.11^{\mathrm{A}}$ \\
\hline C. Nystatin & $49.61 \pm 3.49^{\mathrm{A}}$ & $60.01 \pm 3.39^{\mathrm{A}}$ & $74.54 \pm 2.89$ & $73.52 \pm 1.65$ \\
\hline
\end{tabular}

Percentual reduction of optical density (mean $\pm S . D$.). The label „A, $B, C$ " means significant differences between the effects of the solution by each Candida ssp.
Table 1

MEAN OF PERCENTUAL REDUCTION OF OPTICAL DENSITY BY EACH CANDIDA SUBSPECIES IN THE EXAMINED THERAPEUTIC EFFECT 


\begin{tabular}{|c|c|c|c|c|}
\hline \multirow{2}{*}{ Solution } & \multicolumn{4}{|c|}{ Percentual reduction of OD (\%) } \\
\hline & C.albicans & C. parapsilosis & C. tropicalis & C. glabrata \\
\hline A. Corsodyl & $86.13 \pm 1.17^{\mathrm{C}}$ & $55.20 \pm 2.04^{\mathrm{C}}$ & $90.93 \pm 0.56^{\mathrm{C}}$ & $66.12 \pm 4.15^{\mathrm{C}}$ \\
\hline B. Cervitec Plus & $96.01 \pm 0.21$ & $94.69 \pm 0.63$ & $92.87 \pm 0.51$ & $89.53 \pm 0.38$ \\
\hline C. Nystatin & $97.98 \pm 0.59 \mathrm{~A}$ & $97.13 \pm 0.60^{A}$ & $98.29 \pm 0.015^{A}$ & $97.21 \pm 0.51^{\mathrm{A}}$ \\
\hline
\end{tabular}

Table 2

MEAN OF PERCENTUAL REDUCTION OF OPTICAL DENSITY BY EACH CANDIDA SUBSPECIES IN

THE PREVENTIVE EFFECT EXAMINATION

Percentual reduction of optical density (mean \pm S.D.). The label " $A, B, C$ " means significant differences between the effects of the solution by each Candida ssp.

effectiveness of Corsodyl ${ }^{\circledR}$ was significantly higher compared to Cervitec Plus ${ }^{\circledR}$, but we didn't find statistically important difference between Nystatin and Cervitec Plus ${ }^{\circledR}$ in each of Candida species.

In relation to C. tropicalis and C. glabrata Corsodyl ${ }^{\circledR}$ was followed by Nystatin and Cervitec Plus ${ }^{\circledR}$ and the results of Corsody ${ }^{\circledR}$ and Cervitec Plus ${ }^{\circledR}$ compared with each other showed statistically significant difference $(p \leq 0.05)$.

Table 2 shows the perceptual reduction of each Candida spp. on the biofilm in preventive effect examination. In each case the OD reduction was higher than $55 \%$ and the most effective preventive product for each examined Candida species was the Nystatin, followed by Cervitec Plus $^{\circledR}$ and Corsodyl ${ }^{\circledR}$. In case of Nystatin the mean OD reduction was found between $97.13 \%$ and $98.29 \%$, and it was similar to the OD reduction using Cervitec Plus ${ }^{\circledR}$, where we found $89.53-96.01 \%$. In all Candida groups, OD reduction was significantly higher in case of Nystatin than Corsody ${ }^{\circledR}(p \leq 0.05)$. Cervitec plus ${ }^{\circledR}$ showed nearly the same values than Nystatin, although the differences were not statistically different.

The Candida-associated denture stomatitis is the most common form of oral fungal infections that is quite common in elderly denture wearers [6]. The fitting surface of the acrylic dentures is a good reservoir for oral plaque (including fungi). It has been demonstrated that the adherence of Candida species, (particularly C. albicans), to acrylic surfaces plays an initial role in the pathogenesis of denture stomatitis. Parvinen et al. [22] showed that there is a higher salivary yeast count in denture wearers compared with individuals without removable denture. Furthermore Olsen [23] has shown in 78-100\% of cases presence of Candida species in the oral cavity of full-denture wearing patients with denture-stomatitis, compared with non-denture wearing individuals (30-60\%).

Numerous studies had shown a positive correlation between epithelial dysplasia, oral squamous cell carcinoma and elevated level of Candida species in the oral cavity [24-27]. It had been demonstrated that oral candidosis may play a significant role in malignant transformation generally. On the other hand, the global pathomechanism of dysplasia and malignancy caused by Candida species is still unclear, however, some aspects had already been explored. Marttila et al [28], reported an increased level of carcinogenic acetaldehyde produced by C. albicans in patients with oral squamous cell carcinoma (OSCC) and $C$. albicans can produce nitrosamine molecules, playing important role in malignant transformation also [29]. Unfortunately, the incidence and mortality of OSCC in Europe are the highest in Hungary, approximately 1500 deaths, and 3000 newly diagnosed OSCC is reported per year. The most important role in primary prevention of OSCC is informing the patients about oral cancer and elimination of each possible risk factor play. The $\mathrm{CHX}$ has a wide antimicrobial spectrum including fungi. The cationic biguanid chlorhexidine molecule is able to bind to epithel cells and other negative charged surfaces [30].

The most important antifungal mechanism of $\mathrm{CHX}$ is the coagulation of nucleoproteins and destruction of cell 2810 walls [29]. There are different $\mathrm{CHX}$ containing products such as solutions, gels and varnishes, and use of SRVs is increasing in preventive dentistry. The antifungal effect of such varnishes is poorly examined. However, according to a previous study, the combined use of Fluor Protector ${ }^{\circledR}$ and Cervitec Plus ${ }^{\circledR}$ varnish exhibited the most excellent antibacterial effect on Streptococcus mutans and Streptococcus sobrinus biofilms compared to the controls [31].

In this study, we have investigated the preventive and therapeutic effect of Cervitec Plus ${ }^{\circledR}$ varnish compared with Corsodyl mouthwash on artificial biofilm of $C$. albicans, $C$. parasilosis, C. tropicalis and C. glabarta. To quantify the efficacy of the tested products, XTT-assay was used. The $\mathrm{XTT}$ test is a colorimetric tetrazolium assay of cellular viability.

During the metabolic activity, XTT is converted to water soluble colored formazan, which is easily measurable in the cellular supernatants, and drug susceptibility can be tested without the destruction of the biofilm structure. On the other hand, this method allows investigation of the intact biofilm also. These advantages render the XTT method common in the studies of fungal growth and drug susceptibility.

We found a measurable percentage reduction of Candida biofilms after and before the use of Nystatin and CHX, which is in contrast with results of Chandra et al, who concluded that in vitro Candida biofilms have a high resistance to antifungal agents [33].

Machado et al. [15] investigated seven chlorhexidine containing mouthrinses in various concentrations $(0.06 \%$ $1 \%$ ) on artificial Candida spp. biofilms ( $C$. albicans, $C$. parapsilosis, C. krusei, C. glabrata and C. tropicalis). According to their findings, the examined $\mathrm{CHX}$ solutions were suitable to reduce the viable cells of Candida spp. biofilm. They concluded that the $\mathrm{CHX}$ is able to treat adequately oral candidosis. This report confirms of results of our investigation regarding the therapeutic effect of $\mathrm{CHX}$ products. The antifungal effect of a CHX contain SRV had been investigated poorly before.

In this study we found, that Cervitec Plus ${ }^{\circledR}$ can reduce the Candida biofilm both as a therapeutic and preventive agent, except in case of the therapeutic examination against $C$. glabrata. In the trial of preventive efficacy a higher percentual reduction of Candida biofilms was then in the case of therapeutic examination.

Candida albicans, frequently encountered bacteria in the mouth of elderly patients with different acrylic prosthetics, is the adhering by their filaments on the acrylic surface [34].

Pusateri et al. [14] examined the preventive effect of human saliva proteins (Hst 5 histatin and hBD-3 defensin) compared to $0.12 \% \mathrm{CHX}$ containing solution against $C$. albicans biofilm cells on denture acrylic surface. They measured the reduction of $C$. albicans biofilm after $1 \mathrm{~h}, 24 \mathrm{~h}$, $48 \mathrm{~h}$ and $72 \mathrm{~h}$. This study reported that Hst 5 histatin and CHX solution inhibit effectively the biofilm growth of $C$. albicans and these are possible preventive agent against candida associated oral candidosis. 
Because of the adhesive capability of Cervitec Plus ${ }^{\circledR}$ to acrylate surface, this study inspired us to examine the preventive efficacy of Cervitec Plus ${ }^{\circledR}$ on Candida biofilms in vitro.

\section{Conclusions}

Nowadays, the incidence of Candida associated denture stomatitis is an even frequent oral disease, especially in elderly and immune compromised patients. According to our findings, the $\mathrm{CHX}$ containing mouthwashes are proper alternatives to conventional antifungal agents in the treatment of oral candidosis. In the case of denture stomatitis, the replacement of the Candida infected dentures is often necessary. We found, that the Cervitec Plus $^{\circledR}$, (a CHX containing varnish) is able to prevent the Candida reinfection of the new ly created dentures.

Because of the disadvantageous side-effects of Nystatin (including mouth irritation, diarrhoea, nausea, vomiting, stomach upset, rash, skin irritation, or allergic reaction), Cervitec Plus ${ }^{\circledR}$ can be considered as an alternative preventive agent against Candida species and theoretically could play a role in the prevention of OSCC in acrylate denture wearing patients. Further in vitro and in vivo studies are required to investigate the long-term anti-candidal effect of Cervitec Plus ${ }^{\circledR}$.

Acknowledgement:The authors gratefully acknowledge the support of Ivoclar-Vivadent (Schaan, Liechtenstein) providing Cervitec Plus ${ }^{\circledR}$ varnish.

\section{References}

1.RAO, P.K. (2012): Scholarly J ournal of Medicine 2, 26-30

2.GUIDA RA, (1988), Ear Nose Throat J 67, 832-840

3.ABU-ELTEEN KH, ABU-ALTEEN RM (1998), New Microbiol 21, $41-48$ 4.FAU AT, WALKER DM (1980), Arch Oral Biol 25, 1-10

5.LUCAS VS (1993), Community Dent Oral Epidemiol 21, 313-316 6.ODDS FC (1988), Baillière Tindall W.B. Saunders Company(Second Edition), London, Philadelphia, Toronto, Sydney, Tokyo, p. 56 7.CUMMING CG, WIGHT C, BLACKWELL CL, WRAY D (1990), Oral Microbiol Immunol 5, 82-85

8.CRACIUNESCU, M.C., CRACIUNESCU, E.L., SINESCU, C., RUSU, L.C., LICKER, M., HOGEA, E., NEGRUTIU, M.L, Rev. Chim. (Bucharest), 65, no. 9, 2014, p. 1067
9.MATTHIJS S, ADRIAENS PA (2002), J Clin Periodontol 29, 1-8

10.***https://pubchem.ncbi.nIm.nih.gov/compound/ chlorhexidine_digluconate

11.*** http://chemistry.tutorvista.com/biochemistry/antiseptic.html 12.MOSTAJ O MF, EXTERKATE RA, BUIJS MJ, CRIELAARD W, ZAURA E. (2016), Clin Oral Investig. [Epub ahead of print]

13.ELLEPOLA AN, SAMARANAYAKE LP, (1998), Arch Oral Biol 43, 9991007

14.PUSATERI CR, MONACO EA, EDGERTON M, (2009), Arch Oral Biol 54, 588-594

15.MACHADO FC, PORTELA MB, CUNHA AC, SOUZA IPR, SOEARES RMA, CASTRO GFBA. (2010), Rev Odontol UNESP 39, 271-275

16.FERNANDEZ, Y., MOSTAJ O, M., EXTERKATE, R.A., BUIJ S, M.J ., CRIELAARD, W., ZAURA, E. (2016). Clin Oral Investig, 2016 J un 23 [Epub ahead of print]

17.BOSINCEANU, D.N., SANDU, I.G., BOSINCEANU, D.G., FORNA, N.C., Rev. Chim. (Bucharest), 65, no. 4, 2014, p. 466

18.RAMAGE G, RAJENDRAN R, SHERRY L, WILLIAMS C. International Journal of Microbiology. 2012;12:528521

19.*** www.ivoclarvivadent.us/zoolu-website/media/.../Cervitec

20.*** https://www.medicines.org.uk/emc/medicine/23034

21.KUHN DM, BALKIS M, CHANDRA J, MUKHERJEE PK, GHANNOUM MA (2003), J Clin Microbiol 41, 506-508

22.PARVINEN T. (1984): Scand J Dent Res 92, 412-418

23.OLSEN I. (1974), Acta Odontol Scand 32, 329-333

24.ROED-PETERSEN B, RENSTRUP G, PINDBORG JJ (1970), Scand J

Dent Res 78, 323-328

25.BASTIAAN RJ , READE PC (1982), Oral Surg Oral Med Oral Pathol 53, $148-151$

26.BEGGS KT, HOLMES AR, CANNON RD, RICH AM (2004), J Clin Microbiol 42, 2275-2278

27.ALNUAIMI AD, WIESENFELD D, O'BRIEN-SIMPSON NM, REYNOLDS EC, MCCULLOUGH MJ (2015), Oral Oncol 51, 139-145

28.MARTTILA EPB, SANGLARD D, et al. (2013), Mol Oral Microbiol 28, 281-291

29.HOOPER SJ, CREAN SJ (2009), Head Neck 31, 1228-1239

30.VAAHTONIEMI LH (1997), Acta Odontol Scand 55, 277-281

31.ELLEPOLA AN, SAMARANAYAKE LP (2001), Oral Dis 7, 11-17

32.ERDEM AP, SEPET E, KULEKCI G, TROSOLA SC, GUVEN Y (2012), Int J Med Sci 9, 129-136

33.CHANDRA J, KUHN DM, MUKHERJEE PK, HOYER LL, MCCORMICK T, GHANNOUM MA (2001), J Bacteriol 183, 5385-5394

34.EARAR, K., CERGHIZAN, D., SANDU, A.V., MATEI, M.N., LEATA, R., SANDU, I.G., BEJ INARIU, C., COMAN, M., Mat. Plast.,52, no. 4, 2015, p. 487

Manuscript reeived: 3.04 .2017 